\title{
PReS-FINAL-2162: Development of a risk model to predict methotrexate intolerance in juvenile idiopathic arthritis
}

\author{
P Van Dijkhuizen ${ }^{1 *}$, M Bulatovic-Calasan ${ }^{1}$, S Pluijm², M De Rotte ${ }^{3}$, B Vastert ${ }^{1}$, S Kamphuis ${ }^{4}$, R De Jonge ${ }^{3}$, \\ N Wulffraat ${ }^{1}$
}

From 20th Pediatric Rheumatology European Society (PReS) Congress

Ljubljana, Slovenia. 25-29 September 2013

\section{Introduction}

Methotrexate (MTX) is a cornerstone and safe diseasemodifying anti-rheumatic drug (DMARD) in the treatment of juvenile idiopathic arthritis (JIA). Despite its safety, MTX intolerance occurs frequently, leading to non-compliance, thus hampering efficacy and potentially leading to early discontinuation.

\section{Objectives}

The aim of this study was to construct a risk model to predict MTX intolerance.

\section{Methods}

In a large, prospective JIA cohort, potential predictors (clinical variables and single nucleotide polymorphisms) were determined at the time of MTX start. The Methotrexate Intolerance Severity Score was employed to measure MTX intolerance in the first year of treatment. MTX intolerance was most prevalent at 6 or 12 months after MTX start. This was defined as the outcome for the prediction model. The model was developed in 152 patients using multivariate logistic regression analysis. It was subsequently internally validated using bootstrapping.

\section{Results}

The prediction model included the following predictors: JIA subtype, antinuclear antibody, parent/patient assessment of pain, Juvenile Arthritis Disease Activity Score-27, thrombocytes, alanine aminotransferase and creatinine. The model classified $77.5 \%$ of patients correctly, changing to $66.7 \%$ after internal validation by bootstrapping. The prediction model was transformed into a risk score (range 0 -17). At an optimal cut-off of $\geq 6$, sensitivity was $82.0 \%$, specificity $56.1 \%$, positive predictive value was $58.7 \%$ and negative predictive value $80.4 \%$.

\section{Conclusion}

The prediction model combined routine clinical variables and showed good predictive power to detect MTX intolerance. This easy-to-use tool could assist clinicians in identifying patients at risk to develop MTX intolerance. Consequently, clinicians can monitor them closely and intervene timely before MTX intolerance hampers MTX efficacy.

\section{Disclosure of interest}

None declared.

\section{Authors' details}

${ }^{1}$ paediatric immunology, UMC Utrecht, Utrecht, Netherlands. ${ }^{2}$ Paediatric haemato-oncology, Rotterdam, Netherlands. ${ }^{3}$ Clinical Chemistry, Rotterdam, Netherlands. " paediatric immunology, Erasmus University Medical Centre, Rotterdam, Netherlands.

Published: 5 December 2013

doi:10.1186/1546-0096-11-S2-P174

Cite this article as: Van Dijkhuizen et al:: PReS-FINAL-2162: Development of a risk model to predict methotrexate intolerance in juvenile idiopathic arthritis. Pediatric Rheumatology 2013 11(Suppl 2):P174.

1paediatric immunology, UMC Utrecht, Utrecht, Netherlands

Full list of author information is available at the end of the article 